\title{
Effect of Loading Rate on Fracture Morphology in a High Strength
}

P. R. Guduru Ductile Steel

\author{
G. Ravichandran \\ Mem ASME \\ Graduate Aeronautical Laboratories, \\ Mail Code 105-50, \\ California Institute of Technology, \\ Pasadena, CA 91125
}

Fracture experiments in a high-strength ductile steel $(2.3 \mathrm{Ni}-1.3 \mathrm{Cr}-0.17 \mathrm{C})$ were conducted under static and dynamic loading conditions in a three-point bend and a one-point bend configurations. A qualitative description of the influence of loading rate on the microscopic features of the fracture surfaces and their role in the fracture initiation process was considered. The fracture surfaces consist of tunneled region and shear lips. The size of the shear lips increases with increasing loading rate and is characterized by microvoids and cell structures. The tunneled region consists of large voids and micro-voids that coalesce by impingement. At high loading rates, localized molten zones are observed at the tunnel-shear lip interface. [DOI: 10.1115/1.1371231]

\section{Introduction}

High strength ductile steels constitute a wide variety of structural steels that find a wide variety of applications involving impact and dynamic loading. Understanding the failure mechanisms under a wide range of loading conditions in these materials would lead not only to better design methods but could also lead to ways of enhancing the desired properties of these steels. The $2.3 \mathrm{Ni}-$ $1.3 \mathrm{Cr}-0.17 \mathrm{C}$ steel has been chosen for this investigation because its failure mechanisms are very similar to and are representative of the class of steels mentioned above. The constitutive properties of this steel can be found in the work of Marchand and Duffy [1] and Zhuang and Ravichandran [2]. Extensive work has been conducted in order to understand the common failure mechanisms in steels under quasistatic loading and their influence on fracture toughness. Low [3] was among the early investigators to discuss the effects of microstructure on ductile fracture mechanisms and especially the role of nucleating particles and the particle-matrix interface strength in the void growth and coalescence process. Cox and Low [4] proposed a ductile fracture mechanism by which voids initiate and grow under hydrostatic tension and coalesce by either impingement with each other or formation of void sheets. Though a significant amount of experimental and analytical work has been devoted to study the role of void initiation, growth and coalescence processes in ductile fracture, the complexity of the processes and the diversity of the material systems necessitates systematic experimental investigations in order to make toughness predictions based on microstructural information. Some of the experimental studies of the conditions leading to the nucleation of voids from inclusions, their growth and coalescence were conducted by Argon et al. [5] and Zurek et al. [6]. Using analytical and numerical approaches, among others, Argon et al. [7] have modeled the void initiation processes. Numerous models to characterize void growth and coalescence have been proposed, see for example, Le Roy et al. [8], Curran et al. [9], Needleman and Tvergaard [10], Faleskog and Shih [11], and Tonks et al. [12]. A comprehensive review of void growth to coalescence modeling of ductile failure can be found in Tvergaard [13].

Using the slip line theory, Rice and Johnson [14] characterized the stress field ahead of a blunting crack and investigated the growth of a void under such a stress field, which led to a criterion for its coalescence with the main crack. McMeeking [15] employed a large deformation finite element analysis of the crack tip region and obtained results that were in agreement with those of

Contributed by the Materials Division for publication in the JOURNAL OF ENGINEERING MATERIALS AND TECHNOLOGY. Manuscript received by the Materials Division March 1, 2000; revised manuscript received November 17, 2000. Associate Editor: A Rajendran.
Rice and Johnson [14]. Most of these studies mentioned above have been limited to investigation of quasi-static fracture. An early attempt to correlate the loading rate and the microstructure was made by Couque et al. [16]. Dynamic tension experiments were performed on circumferentially notched tensile specimens. An attempt was made to characterize the observed microstructure in terms of the percentage of fibrous areas and cleavage areas and relate it to such parameters as grain size, temperature, and loading rate. Godse et al. [17] investigated the micromechanisms of dynamic fracture initiation under extremely high rates of loading in 4340 steel. In recent years much progress has been made through numerical studies to understand ductile crack initiation and growth under dynamic loading conditions, for example, Tvergaard and Needleman [18], Needleman [19], Needleman and Tvergaard [20], and Basu and Narasimhan [21].

In this paper, results obtained from fracture experiments and microstructural characterization of the fractured surfaces were combined to understand the changes in the failure mechanisms as a function of loading rate in $2.3 \mathrm{Ni}-1.3 \mathrm{Cr}-0.17 \mathrm{C}$ steel. A brief description of the steel used in the investigation, the experimental methods used to obtain the fracture parameters, and the microstructural characterization techniques are presented. A detailed description of microstructural observations and fracture mechanisms at different loading rates is given and correlated to the crack tip stress state.

\section{Experimental}

2.1 Material. The steel $(2.3 \mathrm{Ni}-1.3 \mathrm{Cr}-0.17 \mathrm{C})$ used in this study has been manufactured by Lukens Steel Co., Coatesville, PA., in a quenched and tempered condition. The chemical composition of the steel used in this study is given in Table 1. The constitutive characterization of the material has been conducted by testing specimens in compression and tension at different loading rates. The yield stress of this material exhibits rate sensitivity, increasing from $780 \mathrm{MPa}$ at a strain rate of $0.001 / \mathrm{s}$ to $1190 \mathrm{MPa}$ at a strain rate of $7050 / \mathrm{s}$.

2.2 Fracture Experiments. The specimens were tested at three loading rates: quasi-static and dynamic at two different impact speeds $(5 \mathrm{~m} / \mathrm{s}$ and $50 \mathrm{~m} / \mathrm{s})$. Fracture experiments have been conducted in a three-point bend configuration and a one-point

Table 1 Chemical composition (wt percent) for the steel

\begin{tabular}{ccccccccccccc}
\hline $\mathrm{C}$ & $\mathrm{Mn}$ & $\mathrm{Cu}$ & $\mathrm{Si}$ & $\mathrm{Ni}$ & $\mathrm{Cr}$ & $\mathrm{Ti}$ & $\mathrm{V}$ & $\mathrm{S}$ & $\mathrm{P}$ & $\mathrm{Sn}$ & $\mathrm{Ca}$ & $\mathrm{Mo}$ \\
\hline 0.17 & 0.30 & 0.13 & 0.22 & 2.35 & 1.32 & 0.003 & 0.004 & 0.004 & 0.004 & 0.013 & 0.005 & 0.25 \\
\hline
\end{tabular}




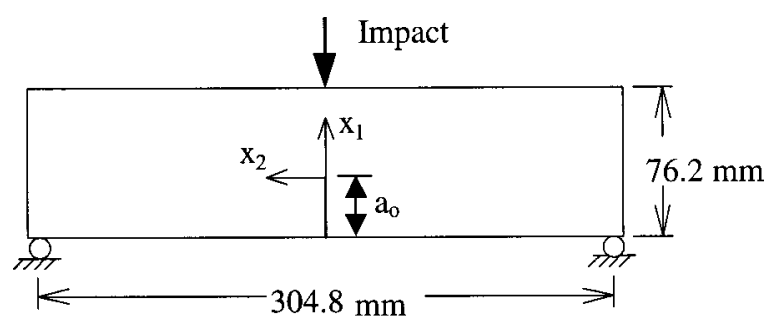

(a)

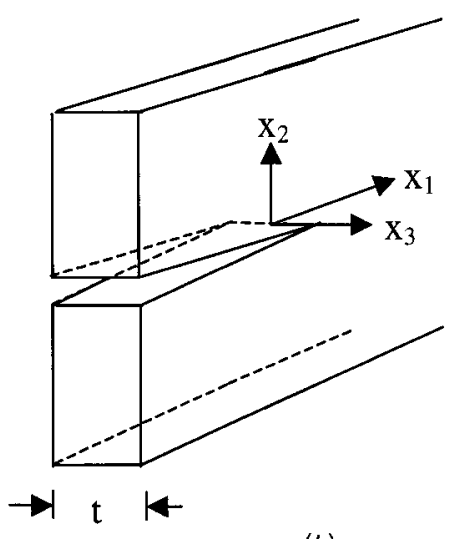

(b)

Fig. 1 (a) Schematic of impact loaded three-point bend precracked steel specimen (thickness, $t=10 \mathrm{~mm}$, precrack length, $a_{0}=30 \mathrm{~mm}$, notch width $=0.25 \mathrm{~mm}$ ); (b) crack tip coordinate system

bend configuration. The specimen geometry and a schematic illustration of the three-point bend loading configuration are shown in Fig. 1. The notch shown was made, parallel to the rolling direction, by electrical discharge machining (EDM), followed by fatigue precracking which extended the notch by $2 \mathrm{~mm}$. Quasistatic experiments were conducted using a servo-hydraulic material testing system.

A medium rate of dynamic loading was achieved by impacting the specimen under a Dynatup 8100A drop weight tower with tup mass of $200 \mathrm{~kg}$ at $5 \mathrm{~m} / \mathrm{s}$ impact velocity. Higher dynamic loading rate was obtained in a specimen impacted with a steel projectile at $50 \mathrm{~m} / \mathrm{s}$ using a gas gun in a one-point bend configuration. Here, one point bend configuration was used because at such high impact speeds, inertial effects are sufficient to initiate failure and no supports are required. This greatly simplifies the experimental setup. In the current experimental geometry, with the crack length to specimen width ratio used, the stress/strain field in front of the crack tip is known to be governed by the $J$ integral through the HRR singular field $[22,23]$. Hence a characterization of the crack tip loading and its resistance to initiation is sought in terms of the $J$ integral. Dynamic $J$ integral was calculated by measuring the evolution of the crack opening displacement (COD) and by measuring the temperature rise ahead of the crack tip. High-speed photography was used to image the deforming crack to measure the COD and a high-speed infrared detector was used to measure the temperature rise. The details of the experimental procedure and data analysis can be found in the work of Guduru et al. [24].

2.3 Microscopy. Samples for microscopic examination were prepared from selected fracture specimens. Samples from the crack initiation area were cut using a diamond saw and were gold coated for better resolution. The samples were examined in a Cambridge 360 scanning electron microscope (SEM). The microscope was equipped with an X-ray energy dispersive spectroscopy (EDS) system which is used for chemical analysis. All chemical analyses were conducted on uncoated samples.

\section{Results}

3.1 Fracture Experiments. Four specimens at each loading rate (the quasi-static and the two dynamic rates) were tested, and the results were found to be highly repeatable. Table 2 summarizes the average values of the loading rates $(\dot{J})$ and the critical value of the $J$ integral $\left(J_{c}\right)$ obtained under the three loading conditions. There is a substantial increase in the toughness at higher

Table 2 Critical value of $J$ integral, $J_{c}$, as a function of loading rate, $j$

\begin{tabular}{cc}
\hline$j\left(\mathrm{kNm}^{-1} \mathrm{~s}^{-1}\right)$ & $J_{c}\left(\mathrm{kNm}^{-1}\right)$ \\
\hline 10 & $1,080( \pm 5 \%)$ \\
$1.5 \times 10^{6}$ & $1,750( \pm 12 \%)$ \\
$22.4 \times 10^{6}$ & $2,230( \pm 12 \%)$
\end{tabular}
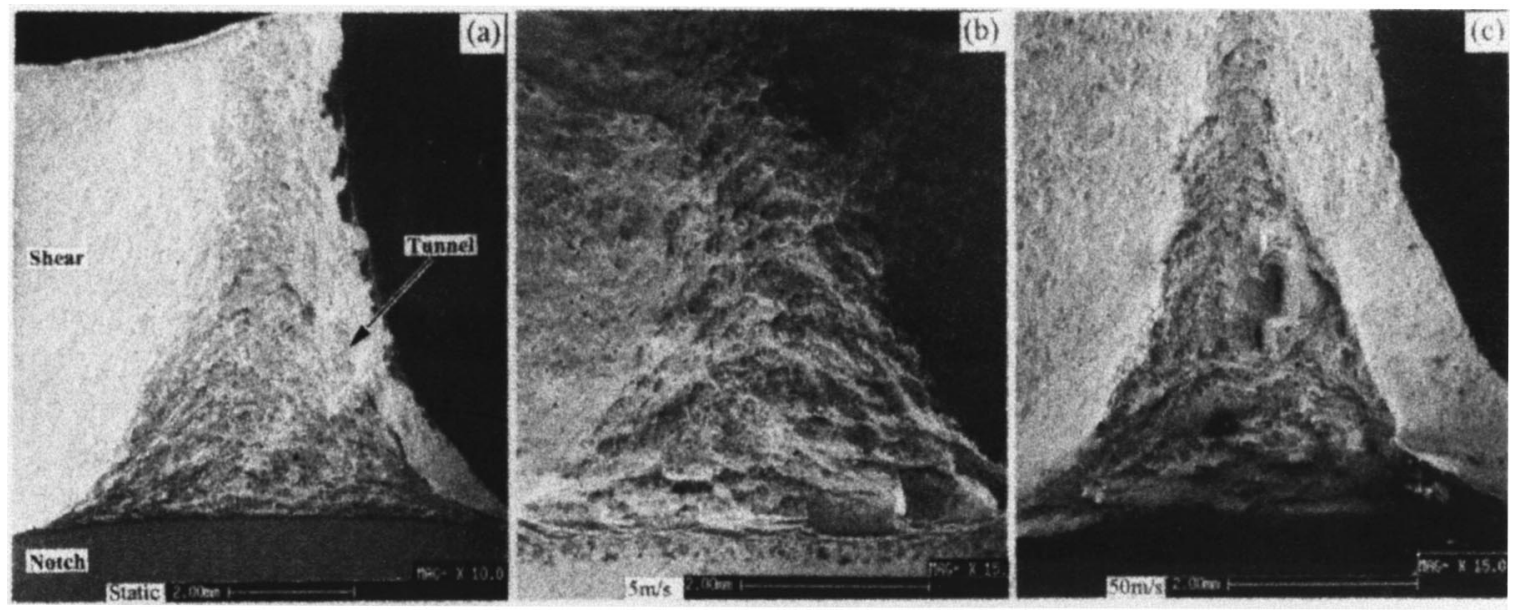

Fig. 2 Comparison of tunneled regions of the fracture surfaces of $2.3 \mathrm{Ni}-1.3 \mathrm{Cr}-0.17 \mathrm{C}$ steel under (a) quasi-static, (b) dynamic loading by drop weight ( $5 \mathrm{~m} / \mathrm{s})$, and (c) dynamic loading by impact using gas gun ( $50 \mathrm{~m} / \mathrm{s}$ ) conditions 
loading rates. Such a trend could be expected from the rate sensitive behavior of yield stress, coupled with inertial effects.

3.2 Microscopy. A low magnification SEM image of the fractured surface of the samples (plane $x_{1}, x_{3}$ in the Fig. 1(b)) is shown in Fig. 2 and reveals the crack initiation region. The prefatigue crack is clearly visible in the lower part of the micrographs. The tunneling region, having a triangular shape, starts from the prefatigue crack and is flanked on the sides by shear lips. The dark flat area on the right side of both micrographs is a saw cut of the shear lip that was made during SEM sample preparation in order to reduce the height of the sample. Figure 2(a) was taken from a quasi-statically loaded $\left(j=10 \mathrm{kN} \mathrm{m}^{-1} \mathrm{~s}^{-1}\right)$ specimen
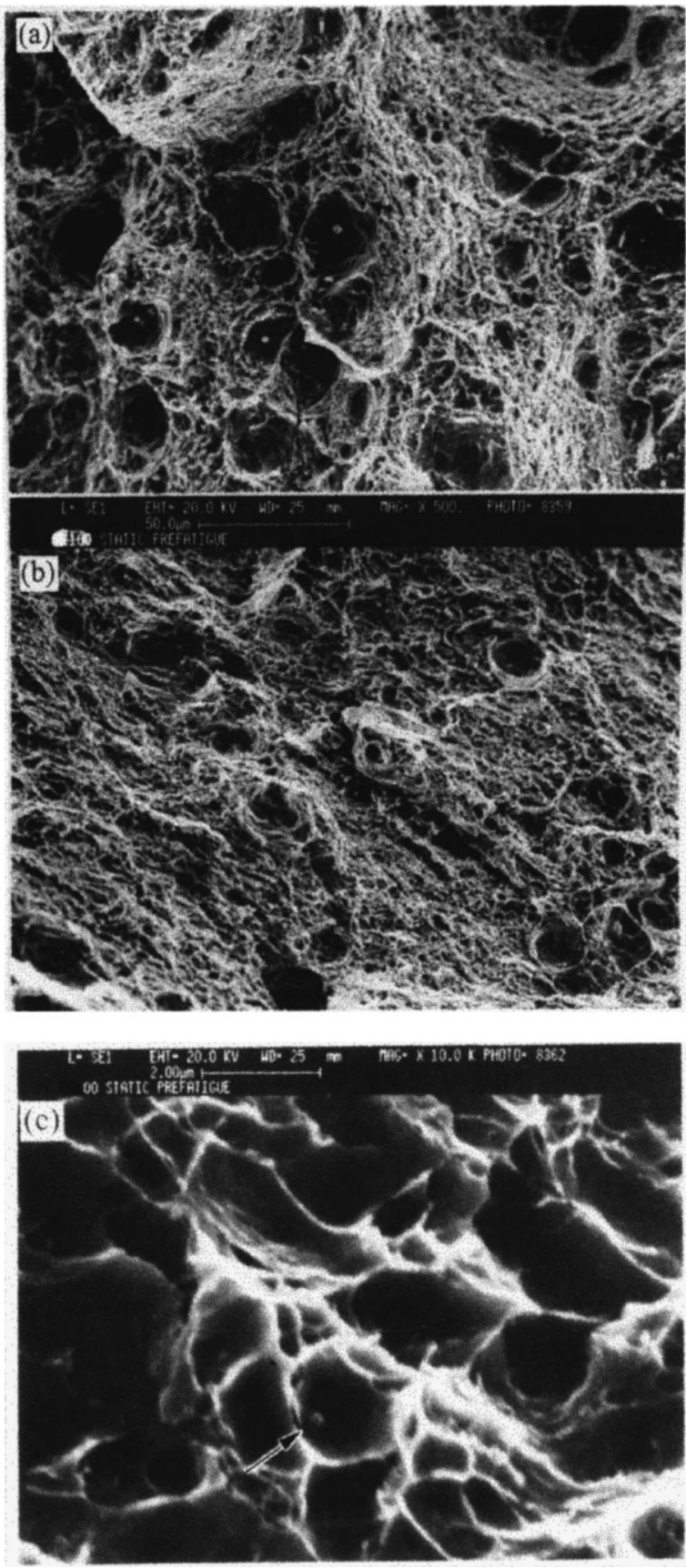

Fig. 3 SEM micrographs of void structure in (a) tunneled area and $(b)$ shear lip region. (c) Is a high magnification image of the shear region showing a $0.1 \mu \mathrm{m}$ size particle (arrowed) that nucleated a microvoid. while Figs. 2(b) and 2(c) were taken from dynamically loaded samples, loaded at a rate of $\dot{J}=1.5 \times 10^{6} \mathrm{kN} \mathrm{m}^{-1} \mathrm{~s}^{-1}$ and $\dot{J}$ $=22.4 \times 10^{6} \mathrm{kN} \mathrm{m}^{-1} \mathrm{~s}^{-1}$, respectively. Note that Fig. $2(a)$ is at a lower magnification than that of Figs. $2(b)$ and $2(c)$ in order to accommodate a larger area.

The image of the tunneling area in the quasi-static case has a uniform appearance while in the highest rate of dynamic loading case $\left(\dot{J}=22.4 \times 10^{6} \mathrm{kN} \mathrm{m}^{-1} \mathrm{~s}^{-1}\right)$ one sees two extremely large voids close to the centerline and a narrow band just ahead of the prefatigue crack. The size of the tunneled region decreases with increase in the loading rate. In the quasi-static sample in Fig. 2(a), it is substantially (almost 50 percent) larger than the same region in the highest loading rate sample in Fig. 2(c).

Higher magnification image of the tunneled area from a high rate fractured sample is shown in Fig. 3(a). Though the void size distribution is different at each rate, this is representative of the morphologies of the tunneled region for all three loading rates. The fracture surface has a dimpled morphology showing large voids in the range of $10-50 \mu \mathrm{m}$. In many of these voids the nucleating inclusions, about $1 \mu \mathrm{m}$ in diameter are visible. The area between the big voids consists of a fine structure of cancellous voids and cells. Fig. 3(b) is an image from the shear lip region and mainly consists of micro-voids and cell structures but still a few big voids are noticeable. This is a typical ductile shear fracture surface. Fig. 3(c) is a high magnification image of the shear region showing microvoids and the cell structures. In this figure, a $0.1 \mu \mathrm{m}$ size particle (arrowed) that nucleated a microvoid can be seen.

Just ahead of the prefatigue crack and before the well developed tunneled zone, a region adjacent to the notch about $200 \mu \mathrm{m}$ wide with cancellous voids, can be observed in the dynamic cases and is shown in Fig. 4. This micrograph is an enlargement of the

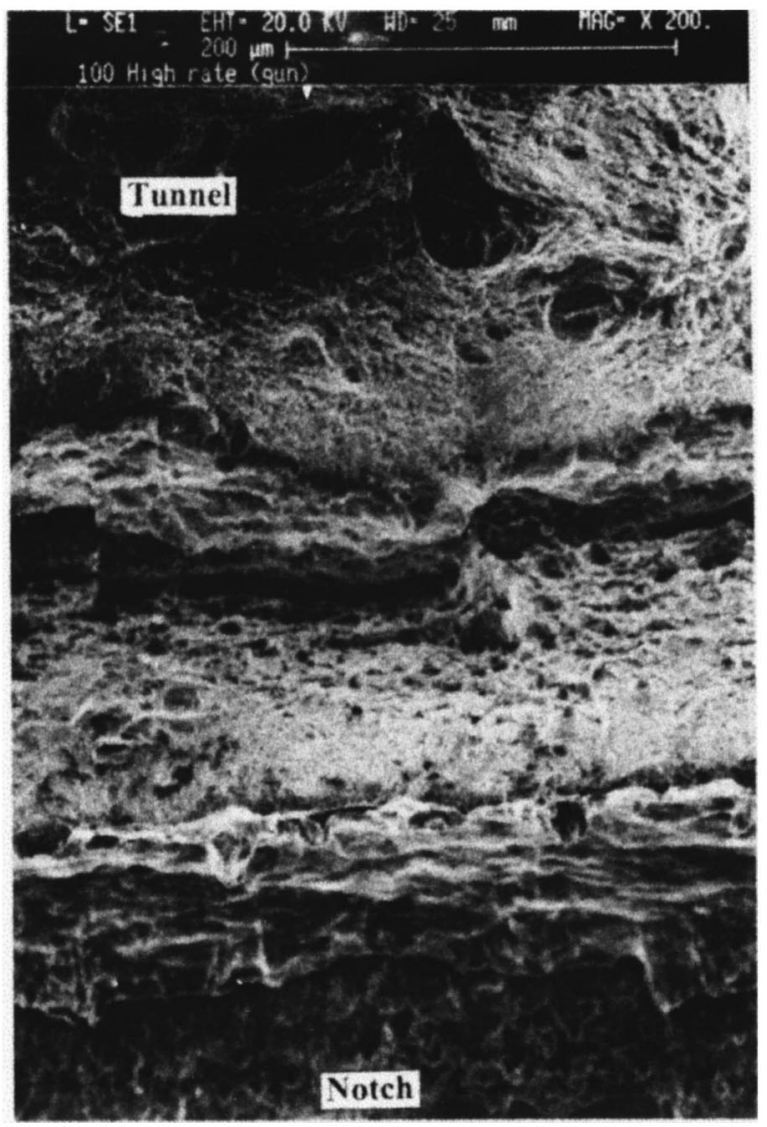

Fig. 4 Micrograph of shear dominated area at the crack initiation site 

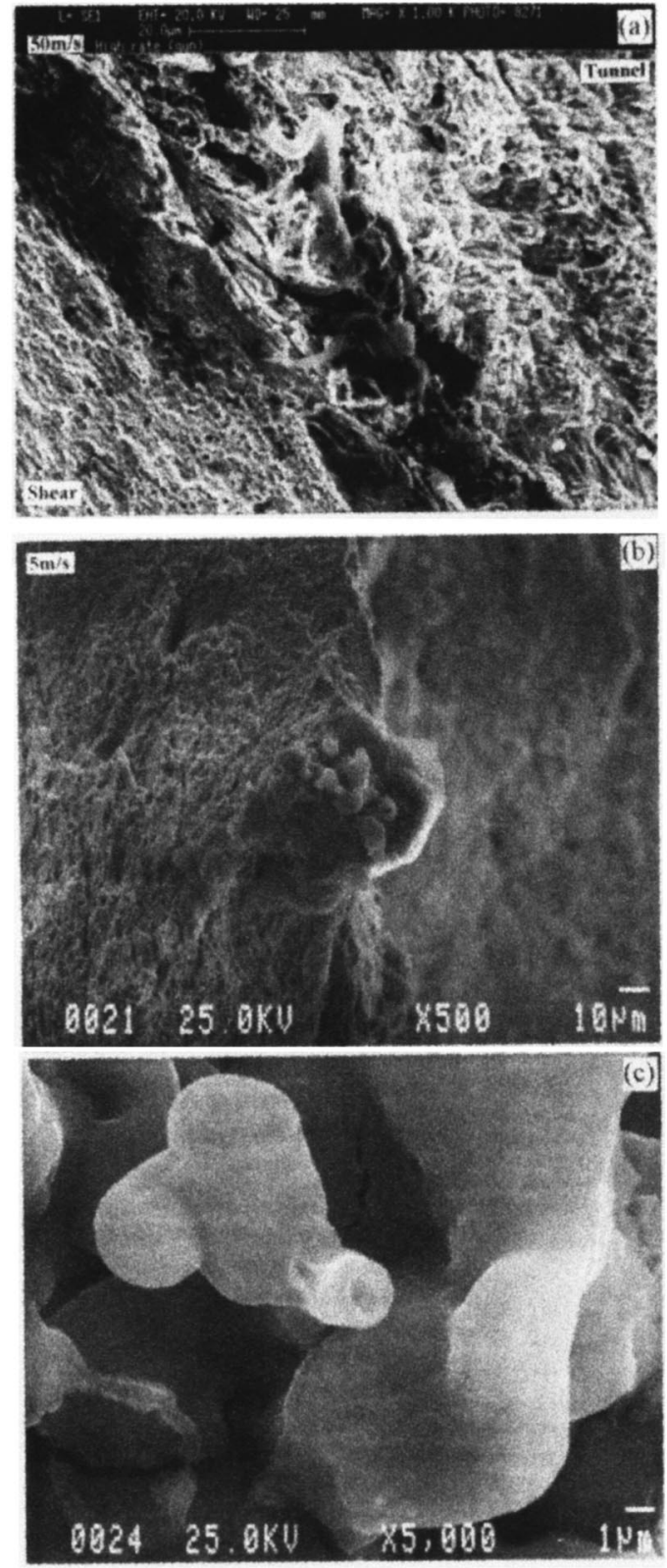

Fig. 5 Molten zones at the tunnel-shear lip interface: (a) 50 $\mathrm{m} / \mathrm{s}$ impact experiment, (b) $5 \mathrm{~m} / \mathrm{s}$ impact experiment and (c) 5 $\mathrm{m} / \mathrm{s}$ impact experiment, at higher magnification.

boundary between the prefatigued crack (bottom of the Fig. 2(c)) and the tunneled region.

The boundary between the tunneled region and the shear lips of the specimen fractured at the highest dynamic loading rate $(j$ $=22.4 \times 10^{6} \mathrm{kN} \mathrm{m}^{-1} \mathrm{~s}^{-1}$ ) is shown in Fig. 5(a). On the right-hand side of the micrograph is the tunneled area and on the left-hand side is the shear lip. The interface between the tunneled and the shear lip regions is highly localized in the form of shear deformed voided bands with evidence of local melting in the material. The molten droplets have been smeared indicating large shear strains in the direction of crack propagation. The same phenomenon was also observed in the sample fractured at the medium rate (drop tower, $\dot{J}=1.5 \times 10^{6} \mathrm{kN} \mathrm{m}^{-1} \mathrm{~s}^{-1}$ ) but was absent in the quasistatically fractured specimens. Figure $5(b)$ was taken from the sample fractured at the medium rate and shows the interface between the two regions (tunnel and shear lip) and a localized mol- ten area within the interface. Figure 5(c) shows an enhanced view of this area at a higher magnification revealing droplets of molten and re-solidified material.

\section{Discussion}

The fracture surface in $2.3 \mathrm{Ni}-1.3 \mathrm{Cr}-0.17 \mathrm{C}$ steel has a dimpled morphology near the middle of the specimen (tunneled area). This region is flanked by areas of shear failure, near the specimen free surfaces (shear lips), which are characteristic features of ductile fracture. Microscopic observation of the fracture surface reveals that the mechanisms of fracture can be classified into the following categories.

(a) The material contains a dual size population of particles, of sizes about $1 \mu \mathrm{m}$ and $0.1 \mu \mathrm{m}$. The tunneled area consists of large voids, that initiate at nucleating particles $1-2 \mu \mathrm{m}$ in size and grow to $10-50 \mu \mathrm{m}$ in diameter. They dominate the fracture surface in the tunneled region, which is approximately triangular in shape (Fig. 2). The nucleating particles can be seen in some of the voids. Other voids do not contain the nucleating particles because either these particles were separated from the specimen during fracture or they are adhered to the voids on the other half of the specimen.

(b) The shear lips and the areas between the large voids in the tunneled region are filled with two features, micro-voids and cell structures. The micro-voids are approximately $1 \mu \mathrm{m}$ in size and are initiated at nucleating particles, about $0.1 \mu \mathrm{m}$ in size. On the other hand, it appears that the other feature, i.e., the cell structure, which is characterized by distinct corners, is similar in size and has no nucleating particle population.

Clearly, the magnitude and the distribution of the stress components ahead of the deforming crack tip determine the relative extent of the above two failure mechanisms. A number of investigations have been conducted to determine the state of stress ahead of a blunting crack tip during deformation (Rice and Johnson [14], McMeeking [15]). Distribution of the hydrostatic stress ahead of the blunted crack tip, which is one of the factors that drive void initiation and growth, is shown schematically for two different values of $J$ in Fig. 6(a). The coordinate system used

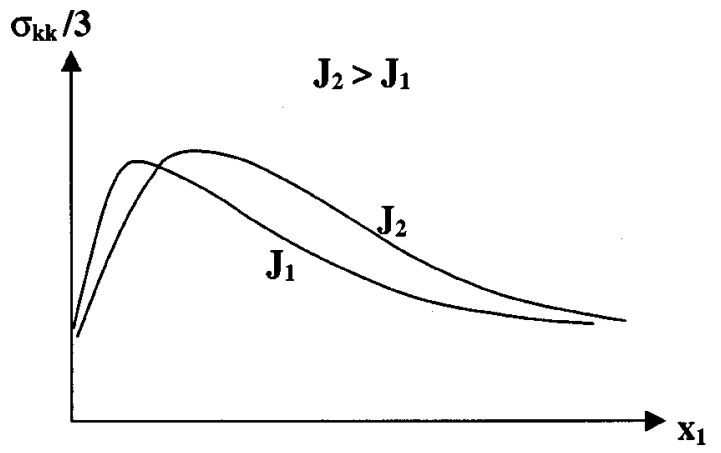

(a)

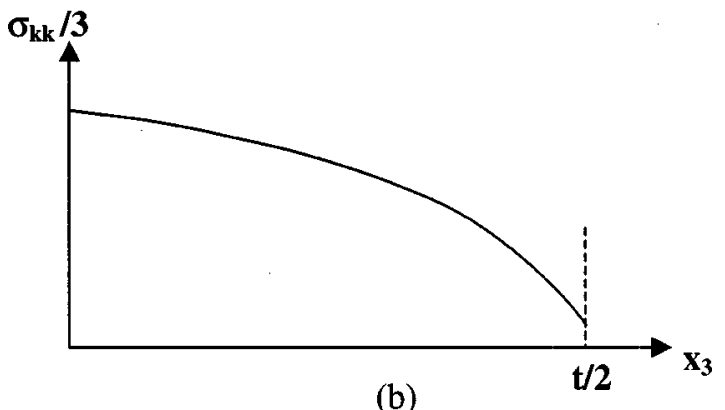

Fig. 6 Schematic illustration of hydrostatic stress $\left(\sigma_{\mathrm{kk}} / 3\right)$ distribution, (a) along $x_{1}$ axis and (b) in the thickness direction 
is shown in Fig. 1(b). This shows that the maximum driving force for the nucleation and growth of voids is at a distance ahead of the blunted crack tip. The location of maximum stress depends on the value of the applied $J$ integral. Also, the traction free condition of the free surface on the sides, i.e., $x_{3}= \pm t / 2$, where $t$ is the specimen thickness, gives rise to a hydrostatic stress distribution in the thickness direction as illustrated in Fig. 6(b) (Nakamura and Parks [25], Narasimhan et al. [26]). This indicates that void growth has the highest driving force near the mid-plane of the specimen, giving rise to extensive void growth there, leading to the formation of tunneled area.

As the porosity increases, the tunneled region loses some of its ability to carry load in the $x_{2}$ direction and the ligaments between the tunneled region and the two free surfaces $\left(x_{3}= \pm t / 2\right)$ on the sides begin to carry more load and accumulate plastic strain in shear. Eventually they fail along the maximum shear stress planes ( $\pm 45 \mathrm{deg})$. These shear failure areas consist of microvoids of size $\sim 1 \mu \mathrm{m}$ that originate at inclusions of $\sim 0.1 \mu \mathrm{m}$ size and the characteristic cell structures, very similar to the areas between the large voids in the tunneled region. This leads one to conjecture that the accumulated shear plastic strains in the shear lips initiate micro-voids at the $0.1 \mu \mathrm{m}$ particles, which grow and impinge upon each other, leading to material separation in the shear lips.

Examining Fig. 2, one could notice the difference in the void size distribution between the quasi-static $\left(\dot{J}=10 \mathrm{kN} \mathrm{m}^{-1} \mathrm{~s}^{-1}\right)$, medium rate (drop tower, $\dot{J}=1.5 \times 10^{6} \mathrm{kN} \mathrm{m}^{-1} \mathrm{~s}^{-1}$ ), and the high rate (gas gun, $\dot{J}=22.4 \times 10^{6} \mathrm{kN} \mathrm{m}^{-1} \mathrm{~s}^{-1}$ ) cases. In the quasistatic case, the void size distribution in the tunneled area appears to be more or less uniform. In the high rate case, the tunneled area is highly nonuniform, with a large variation in void sizes. Some of the voids are of the order of a millimeter, shaped like a coin. The medium rate case is somewhere in between. Under quasistatic loading conditions, void growth takes place under equilibrium conditions. Because of the lack of equilibrium under dynamic conditions, coupled with the high growth rate of the initiated voids, the voids that are initiated earlier, at the relatively large inclusions, grow rapidly at the expense of those that initiate later, leading to a highly nonuniform void size distribution. As an extreme case of this, the voids that are initiated very early at large inclusions grow to become very large in size, of the order of a millimeter. This is akin to the phenomenon of cavitation in elastic-plastic solids (Chung et al. [27]) where small voids grow unboundedly when the far-field hydrostatic stress reaches a critical value, in the absence of competing voids in the neighborhood. The rapid void growth phase is followed by the void coalescence process, which involves extensive plastic strains, leading to material separation. These normal strains result in severe stretching in the $x_{2}$ direction and contraction in the $x_{3}$ direction resulting in the coin like shape of these very large sized voids.

Careful observation of the fracture surface in the dynamic cases reveals that just ahead of the prefatigued crack surface and before the well developed tunnel zone, there is a narrow layer that runs along the specimen thickness (Fig. 4). Its width is about $200 \mu \mathrm{m}$ in the $5 \mathrm{~m} / \mathrm{s}$ impact experiment and it is about $300 \mu \mathrm{m}$ in the 50 $\mathrm{m} / \mathrm{s}$ case. Such a layer is almost nonexistent in the static experiment. The microvoid population and the cell structures observed in this layer are similar to those that dominate the shear lip region, suggesting a shear failure. It is well established from the work of Rice and Johnson [14] and McMeeking [15] that the maximum hydrostatic stress occurs at a distance ahead of the crack tip (Fig. 6). From the work of Rice and Johnson [14], for the current material properties, this distance is given by $x=0.005\left(J E / \sigma_{0}^{2}\right)$. For the three loading rates considered in this study, these distances, for the corresponding critical values of $J$, are $0.32 \mathrm{~mm}, 0.53 \mathrm{~mm}$ and $0.67 \mathrm{~mm}$, respectively. This results in a narrow ligament between the voided area and the main crack, which is subjected to tensile stretching, leading to failure by shear along the maximum shear stress planes. This is schematically illustrated in Fig. 7(a).

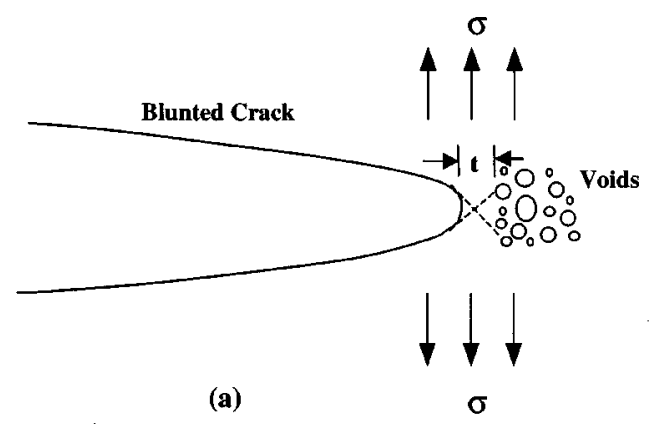

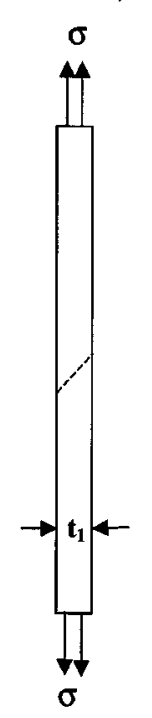

(b)

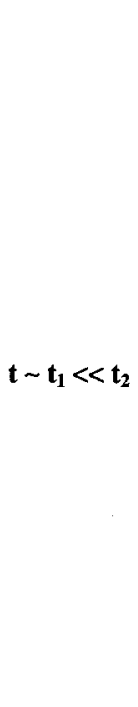

(c)

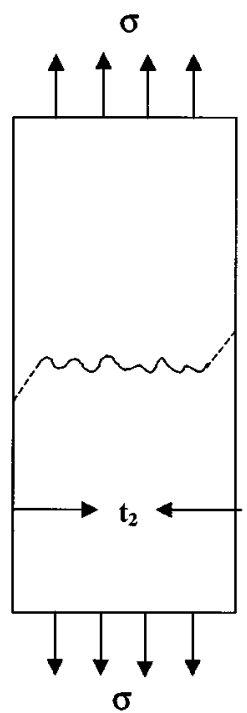

Fig. 7 Schematic illustration of shear failure of the ligament at the crack tip: (a) ligament between the blunted crack tip and the voided region subjected to tensile stretching. Illustration of failure modes in plate geometries: $(b)$ a thin strip subjected to tension undergoes shear failure along $45 \mathrm{deg}$ planes and (c) a thick plate subjected to tension undergoes shear failure at the edges and fracture by void growth and coalescence in the interior.

This situation is analogous to the stretching of a thin strip of metal that results in failure along the 45 deg shear planes as shown in Fig. 7(b). This contrasts with the stretching of a thick rod, that results in a cup and cone fracture, with shear fracture at the edges and void fracture at the center (Fig. 7(c)). The dashed lines in Fig. $7(a, b, c)$ are the planes of shear failure. Further, the width of this ligament depends on the critical stress required for void nucleation and growth. For a given set of critical conditions, this width increases with the critical value of $J$ suggesting that the ligament becomes more pronounced at higher loading rates, which is consistent with the observations (Fig. 2). Though this does not provide a quantitative explanation for the exact size of the width, it qualitatively accounts for the observed trends.

One of the important differences between the static and the dynamic loading is the critical value of the $J$ integral. It can be seen from Table 2 that at the highest loading rate considered, its value is almost twice that at the quasi-static loading case. This has implications for the size of the plastic zone $\left(r_{p}\right)$ at the time of crack initiation. Crack tip plastic zone size scales with the ratio $J / \sigma_{0}$, where $\sigma_{0}$ is the yield stress. From Table 2, $J_{c}^{\text {dynamic }}$ $>J_{c}^{\text {static }}$. Assuming that the Hutchinson [22], Rice and Rosengren [23] (HRR) description for stresses and strains near the crack tip holds good, the strain rate ahead of the crack tip can be written as: 


$$
\dot{\varepsilon}_{i j}=\varepsilon_{0} \frac{1}{\left(\sigma_{0} \varepsilon_{0} I_{n} r\right)^{n / n+1}}\left(\frac{n}{n+1}\right) J^{1 /(n+1)} \dot{J} E_{i j}(n, \theta)
$$

where $(r, \theta)$ are polar coordinates with the origin at the crack tip and $\theta$ measured from the crack line in front of the crack tip. $\varepsilon_{i j}$ are the components of strain, $n, \varepsilon_{0}, \sigma_{0}$, and $I_{n}$ are material constants, $E_{i j}$ are functions of $n$ and $\theta$ and dot denotes time derivative. Using $\varepsilon_{0}=0.004, \sigma_{0}=800 \mathrm{MPa}, n=8$ and $I_{n}=4$ [2], for the highest dynamic case considered, at a distance of a fraction of a millimeter in front of the crack tip, the strain rates can be estimated to be of the order of 100/s. Constitutive data reported in the above reference would also imply that the yield stress $\sigma_{0}^{\text {dynamic }}$ is effectively the same as $\sigma_{0}^{\text {static }}\left(\sigma_{0}^{\text {dynamic }} \approx \sigma_{0}^{\text {static }}\right)$. Thus, the size of the plastic zone under high loading rates is bigger than that quasi-static loading conditions $\left(r_{p}^{\text {dynamic }}>r_{p}^{\text {static }}\right)$. The width of the shear lip is an increasing function of the size of the plastic zone. Hence, one would expect a bigger shear lip under dynamic loading conditions, which also implies a smaller tunnel region. This provides an explanation for the experimentally observed difference in the size of the tunneled area as a function of loading rate, as shown in Fig. 2.

Localized molten zones were observed for the first time at the tunnel-shear lip interface (Fig. 5). The most plausible explanation for the appearance of molten metal during dynamic deformation is deformation trapping in a thin layer at the tunnel-shear lip interface which leads to shear localization in the interface [28]. In experimental studies involving high speed shearing in steels, it was found [29] that shear localization tends to occur when the shearing speed reaches the range of $100-130 \mathrm{~m} / \mathrm{s}$ which was termed as critical impact velocity. In the current study, using highspeed photography, the crack was observed to propagate at a speed of about $100 \mathrm{~m} / \mathrm{s}$ upon initiation. This is also the speed of shearing imposed on the tunnel/shear lip interface. Deformation trapping coupled with moderate thermal conductivity of steel, would lead to very high temperatures at the tunnel-shear lip interface, resulting in localized melting. Evidence of such shear localization can be seen in Fig. 5(a) in the form of molten metal aligned with the direction of crack propagation and the directionally distorted cell-void structure, which surrounds the molten zones.

\section{Concluding Remarks}

Based on the microstructural observations of fracture surfaces, the following conclusions can be made regarding the micromechanisms of failure in high strength ductile steel as a function of loading rate.

1 The fracture surface consists of large voids, microvoids and cell structures. The tunneled area is dominated by the large voids, with the microvoids and the cell structures filling up the space between the large voids. The fracture surface in the shear lips was very similar to the areas between the large voids in the tunneled region.

2 Tunneling appears to occur by the coalescence of dual population (large, $10-50 \mu \mathrm{m}$ and micro, $\sim 1.0 \mu \mathrm{m}$ ) voids by impingement. The shear lips appear to have formed by the initiation, growth and impingement of microvoids.

3 Between the initial crack tip and the well-developed tunneled area, there is a narrow band that fails by shear. This band is most obvious in the dynamic loading cases considered here and becomes less prominent at quasistatic loading rates. The existence of this layer could be explained through the occurrence of the maximum hydrostatic stress not at the crack tip, but at a distance ahead of it. This layer becomes wider at high loading rates because the increase in the critical $J$ integral increases the distance between the crack tip and the location of the maximum hydrostatic stress.
4 Void growth under nonequilibrium conditions results in a non-uniform void population under dynamic conditions. Under extreme conditions, this leads to huge coin shaped voids at millimeter scale.

5 Increase in the critical $J$ integral value with loading rate increases the plastic zone size under dynamic conditions, leading to a wider shear lip and consequently a smaller tunnel area.

6 Localized molten zones were observed for the first time at the tunnel-shear lip interface. This could be explained through the shear localization occurring at the interface as a result of deformation trapping.

\section{Acknowledgments}

The authors acknowledge the support of the Office of Naval Research under grant no. N00014-95-0453 (Dr. G. Yoder, Scientific officer) to the California Institute of Technology.

\section{References}

[1] Marchand, A., and Duffy, J., 1988, “An Experimental Study of the Formation Process of Adiabatic Shear Bands in a Structural Steel,' J. Mech. Phys. Solids, 36, pp. 251-283.

[2] Zhuang, S., and Ravichandran, G., 1998, SM Report No. 98-4, Graduate Aeronautical Laboratories, California Institute of Technology, Pasadena, CA.

[3] Low, J. R., Jr., 1968, "Effects of Microstructure on Fracture Toughness of High Strength Alloys," Eng. Fract. Mech., 1, pp. 47-53.

[4] Cox, T. B., and Low, J. R., Jr., 1974, “An Investigation of the Plastic Fracture of AISI 4340 and 18 Nickel-200 Grade Maraging Steels,' Metall. Trans. A, 5, pp. $1457-1470$.

[5] Argon, A. S., Im, J., and Safoglu, R., 1975, "Cavity Formation from Inclusions in Ductile Fracture,'” Metall. Trans. A, 6, pp. 825-837.

[6] Zurek, A. K., Thissell, W. R., Tonks, D. L., Hixson, R., and Addessio, F., 1997, "Quantification of Damage Evolution for a Micromechanical Model of Ductile Fracture in Spallation of Tantalum,'” J. Phys. IV, C3, pp. 903-908.

[7] Argon, A. S., and Im, J., 1975, "Separation of Second Phase Particles in Spheroidized 1045 Steel, Cu-0.6Pct Cr Alloy and Maraging Steel in Plastic Straining,' Metall. Trans. A, 6, pp. 839-851.

[8] Le Roy, G., Embury, J. D., Edward, G., and Ashby, M. F., 1981, “'A Model of Ductile Fracture Based on the Nucleation and Growth of Voids,' Acta Metall., 29, pp. $1509-1522$

[9] Curran, D. R., Seaman, L., and Shockey, D. A., 1987, 'Dynamic Failure of Solids,', Phys. Rep., 147, pp. 253-388.

[10] Needleman, A., and Tvergaard, V., 1987, “An Analysis Of Ductile Rupture Modes At A Crack Tip,' J. Mech. Phys. Solids, 35, pp. 151-183.

[11] Falskeog, J., and Shih, C. F., 1997, "Micromechanics of Coalescence. 1. Synergistic Effects of Elasticity, Plastic Yielding and Multi-Size-Scale Voids,', J. Mech. Phys. Solids, 45, pp. 21-50.

[12] Tonks, D. L., Thissell, W. R., Zurek, A. K., and Hixson, R., 1997, "'Quantative Analysis of Damage Clustering and Void Linking for Spallation Modeling in Tantalum,"' J. Phys. IV, C3, pp. 841-846.

[13] Tvergaard, V., 1990, "Material Failure By Void Growth To Coalescence," Adv. Appl. Mech., 27, pp. 83-151.

[14] Rice, J. R., and Johnson, M. A., 1970, "'The Role of Large Crack Tip Geometry Changes in Plane Strain Fracture,'” Inelastic Behavior of Solids, McGrawHill, New York, pp. 641-672.

[15] McMeeking, R. M., 1977, “Finite Deformation Analysis of Crack-Tip Opening in Elastic-Plastic Materials and Implications for Fracture,'” J. Mech. Phys. Solids, 25, pp. 357-381.

[16] Couque, H., Asaro, R. J., Duffy, J., and Lee, S. H., 1988, "Correlations of Microstructure with Dynamic and Quasi-Static Fracture in a Plain CarbonSteel,"' Metall. Trans. A, 19, pp. 2179-2206.

[17] Godse, R., Ravichandran, G., and Clifton, R. J., 1989, "Micromechanisms of Dynamic Crack Propagation in an AISI-4340 Steel,' Mater. Sci. Eng., A112, pp. 79-88.

[18] Tvergaard, V., and Needleman, A., 1986, "Effect Of Material Rate Sensitivity On Failure Modes In The Charpy V-Notch Test,"' J. Mech. Phys. Solids, 34, pp. 213-241.

[19] Needleman, A., 1997, "Numerical Modeling Of Crack Growth Under Dynamic Loading Conditions,' Comput. Mech., 19, pp. 463-469.

[20] Needleman, A., and Tvergaard, V., 1998, “Dynamic Crack Growth In A Nonlocal Progressively Cavitating Solid,' Eur. J. Mech. A/Solids, 17, pp. 421438.

[21] Basu, S., and Narasimhan, R., 1999, “A Finite Element Study Of The Effects Of Material Characteristics And Crack Tip Constraint On Dynamic Ductile Fracture Initiation,'” J. Mech. Phys. Solids, 47, pp. 325-350.

[22] Hutchinson, J. W., 1968, "Singular Behavior at the End of a Tensile Crack in a Hardening Material,', J. Mech. Phys. Solids, 16, pp. 13-31.

[23] Rice, J. R., and Rosengren, G. F., 1968, "Plane Strain Deformation Near a Crack Tip in a Power Law Hardening Material,’’ J. Mech. Phys. Solids, 16, pp. $1-12$. 
[24] Guduru, P. R., Singh, R. P., Ravichandran, G., and Rosakis, A. J., 1998, "Dynamic Crack Initiation in Ductile Steels," J. Mech. Phys. Solids, 46, pp. 1997-2016.

[25] Nakamura, T., and Parks, D. M., 1990, “3-Dimensional Crack Front Fields In A Thin Ductile Plate,' J. Mech. Phys. Solids, 38, pp. 787-812.

[26] Narasimhan, R., Rosakis, A. J., and Moran, B., 1992, “A 3-Dimensional Numerical Investigation Of Fracture Initiation By Ductile Failure Mechanisms In A 4340 Steel," Int. J. Fract., 56, pp. 1-24.
[27] Chung, D.-T., Horgan, C. O., and Abeyaratnne, R., 1987, “'A Note on a Bifurcation Problem in Finite Plasticity Related to Void Nucleation,'” Int. J. Solids Struct., 23, pp. 983-988.

[28] Wu, F. H., and Freund, L. B., 1984, "'Deformation Trapping Due To Thermoplastic Instability In One-Dimensional Wave-Propagation,' J. Mech. Phys. Solids, 32, pp. 119-132.

[29] Klepaczko, J. R., 1998, “Remarks on Impact Shearing," J. Mech. Phys. Solids, 46, pp. 2139-2153. 